\title{
The helium star donor channel for the progenitors of type Ia supernovae and their surviving companion stars
}

\author{
Bo Wang ${ }^{*, \dagger}$ and Zhanwen Han*,† \\ ${ }^{*}$ National Astronomical Observatories/Yunnan Observatory, CAS, Kunming, 650011, China; \\ wangbo@ynao.ac.cn, zhanwenhan@ynao.ac.cn \\ ${ }^{\dagger}$ Key Laboratory for the Structure and Evolution of Celestial Objects, CAS, Kunming 650011, China
}

\begin{abstract}
The nature of type Ia supernovae (SNe Ia) is still unclear. Employing Eggleton's stellar evolution code with the optically thick wind assumption, we systematically studied the He star donor channel of SNe Ia, in which a carbon-oxygen white dwarf accretes material from a He main-sequence star or a He subgiant to increase its mass to the Chandrasekhar mass. We mapped out the initial parameters for producing SNe Ia in the orbital period-secondary mass plane for various WD masses from this channel. According to a detailed binary population synthesis approach, we find that this channel can produce SNe Ia with short delay times $(\sim 100 \mathrm{Myr})$ implied by recent observations. We obtained many properties of the surviving companions of this channel after SN explosion, which can be verified by future observations. We also find that the surviving companions from the SN explosion scenario have a high spatial velocity $(>400 \mathrm{~km} / \mathrm{s}$ ), which could be an alternative origin for hypervelocity stars (HVSs), especially for HVSs such as US 708.
\end{abstract}

Keywords: binaries: close, stars: evolution, supernovae: general, white dwarfs PACS: $97.80 . \mathrm{Fk}, 97.10 . \mathrm{Cv}, 97.60 . \mathrm{Bw}, 97.20 . \mathrm{Rp}$

\section{INTRODUCTION}

Type Ia supernovae (SNe Ia) play an important role in astrophysics, especially in the study of cosmic evolution. They have been applied successfully in determining cosmological parameters (e.g., $\Omega_{M}$ and $\left.\Omega_{\Lambda} ;[1,2]\right)$. They are also a key part of our understanding of galactic chemical evolution owing to the main contribution of iron to their host galaxies. It is generally believed that SNe Ia are thermonuclear explosions of carbon-oxygen white dwarfs (CO WDs) in binaries. However, there is still no agreement on the nature of their progenitors [3-6], and no SN Ia progenitor system before the explosion has been conclusively identified [7-9].

Over the past few decades, two families of SN Ia progenitor models have been proposed, i.e., the double-degenerate (DD) and single-degenerate (SD) models. Of these two models, the SD model is widely accepted at present. It is suggested that the DD model, which involves the merger of two CO WDs [10-12], likely leads to an accretioninduced collapse rather than to an SN Ia [13]. For the SD model, the companion could be a main-sequence (MS) star or a slightly evolved subgiant star (WD + MS channel), or a red-giant star (WD + RG channel) [14-26]. Observationally, there is increasing evidence indicating that at least some SNe Ia may come from the SD model [27-30].

Meanwhile, a CO WD may also accrete material from a He star to increase its mass to the Chandrasekhar (Ch) mass, which is known as the He star donor channel. Yoon and Langer [31] followed the evolution of a CO WD + He star system, in which the WD can increase its mass to the Ch mass by accreting material from the He star. It is believed that WD + He star systems are generally originated from intermediate mass binaries, which may explain SNe Ia with short delay times implied by recent observations [32-35].

In this contribution, we aim to investigate SN Ia birthrates and delay times of the He star donor channel and to explore the properties of the surviving companions after SN explosion. In Sect. 2, we describe the numerical code for the binary evolution calculations and the binary evolutionary results. We describe the binary population synthesis (BPS) method and results in Sect. 3. Finally, a discussion is given in Sect. 4.

\section{BINARY EVOLUTION CALCULATIONS}

We use Eggleton's stellar evolution code $[36,37,38]$ to calculate the binary evolutions of WD + He star systems. The code has been updated with the latest input physics over the past three decades [39, 40,41]. Roche lobe overflow 


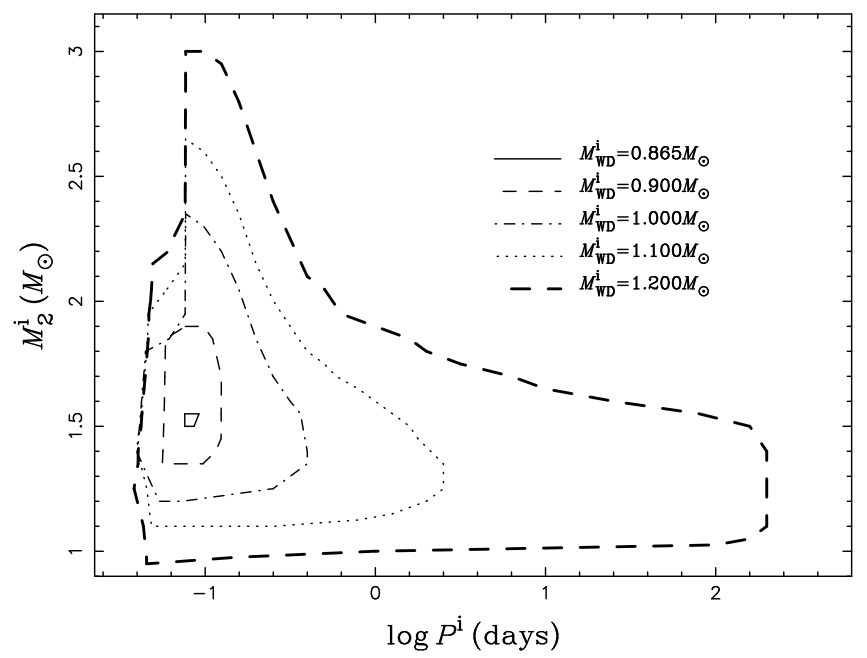

FIGURE 1. Regions in the orbital period-secondary mass plane for WD binaries (at the onset of the RLOF stage) that produce SNe Ia for initial WD masses of $0.865,0.9,1.0,1.1$ and $1.2 M_{\odot}$. (From Wang et al. [45])

(RLOF) is treated within the code described by Han et al. [42]. We set the ratio of mixing length to local pressure scale height, $\alpha=l / H_{\mathrm{p}}$, to be 2.0. The opacity tables are compiled by Chen and Tout [43]. In our calculations, He star models are composed by He abundance $Y=0.98$ and metallicity $Z=0.02$.

Instead of solving stellar structure equations of a WD, we use the optically thick wind model [14] and adopt the prescription of Kato \& Hachisu [44] for the mass accumulation efficiency of He-shell flashes onto the WD. We have calculated about $2600 \mathrm{WD}+\mathrm{He}$ star systems, and obtain a large, dense model grid (for details see [45]). In Fig. 1, we show the contours at the onset of RLOF for producing SNe Ia in the $\log P^{\mathrm{i}}-M_{2}^{\mathrm{i}}$ plane for various WD masses (i.e., $M_{\mathrm{WD}}^{\mathrm{i}}=0.865,0.90,1.0,1.1$ and $\left.1.2 M_{\odot}\right)$, where $P^{\mathrm{i}}$ and $M_{2}^{\mathrm{i}}$ are the orbital period and the mass of the He companion star at the onset of RLOF, respectively. The region almost vanishes for $M_{\mathrm{WD}}^{\mathrm{i}}=0.865 M_{\odot}$, which is then assumed to be the minimum WD mass for producing SNe Ia from this channel. If the parameters of a WD + He star system at the onset of the RLOF are located in the contours, an SN Ia is then assumed to be produced. Thus, these contours can be expediently used in BPS studies to investigate the He star donor channel of SNe Ia. The data points and the interpolation FORTRAN code for these contours can be supplied on request by contacting BW.

\section{BINARY POPULATION SYNTHESIS}

To obtain SN Ia birthrates and delay times of the He star donor channel, we performed a series of Monte Carlo simulations in the BPS study. In the simulation, by using the Hurley's rapid binary evolution code [46, 47], we followed the evolution of $4 \times 10^{7}$ sample binaries from the star formation to the formation of the WD $+\mathrm{He}$ star systems according to the SN Ia production regions (Fig. 1) and three evolutionary channels (i.e., the He star channel, the EAGB channel, and the TPAGB channel; for details see [48]). Here, we adopt the standard energy equations to calculate the output of the common envelop (CE) phase (see [48]).

In the BPS study, the primordial binary samples are generated in the Monte Carlo way and a circular orbit is assumed for all binaries. We adopt the following input for the simulation (for details see [48]). (1) The initial mass function of Miller and Scalo [49] is adopted. (2) The mass-ratio distribution is taken to be constant. (3) The distribution of separations is taken to be constant in $\log a$ for wide binaries, where $a$ is the orbital separation. (4) We simply assume a constant star formation rate (SFR) over the past $15 \mathrm{Gyr}$ or, alternatively, as a delta function, i.e., a single starburst.

In Fig. 2, we show the evolution of SN Ia birthrates with time for the He star donor channel. The left panel represents Galactic birthrates of SNe Ia by adopting $Z=0.02$ and SFR $=5 M_{\odot} \mathrm{yr}^{-1}$. The simulations give Galactic SN Ia birthrates of $\sim 0.3 \times 10^{-3} \mathrm{yr}^{-1}$. The right panel displays the evolution of SN Ia birthrates for a single starburst with a total mass of $10^{11} M_{\odot}$. In this panel, we see that SN Ia explosions occur between $\sim 45 \mathrm{Myr}$ and $\sim 140 \mathrm{Myr}$ after the starburst, which could explain SNe Ia with short delay times implied by recent observations [32-35].

The companion in the SD model would survive in the SN explosion and potentially be identifiable [50, 51]. We 

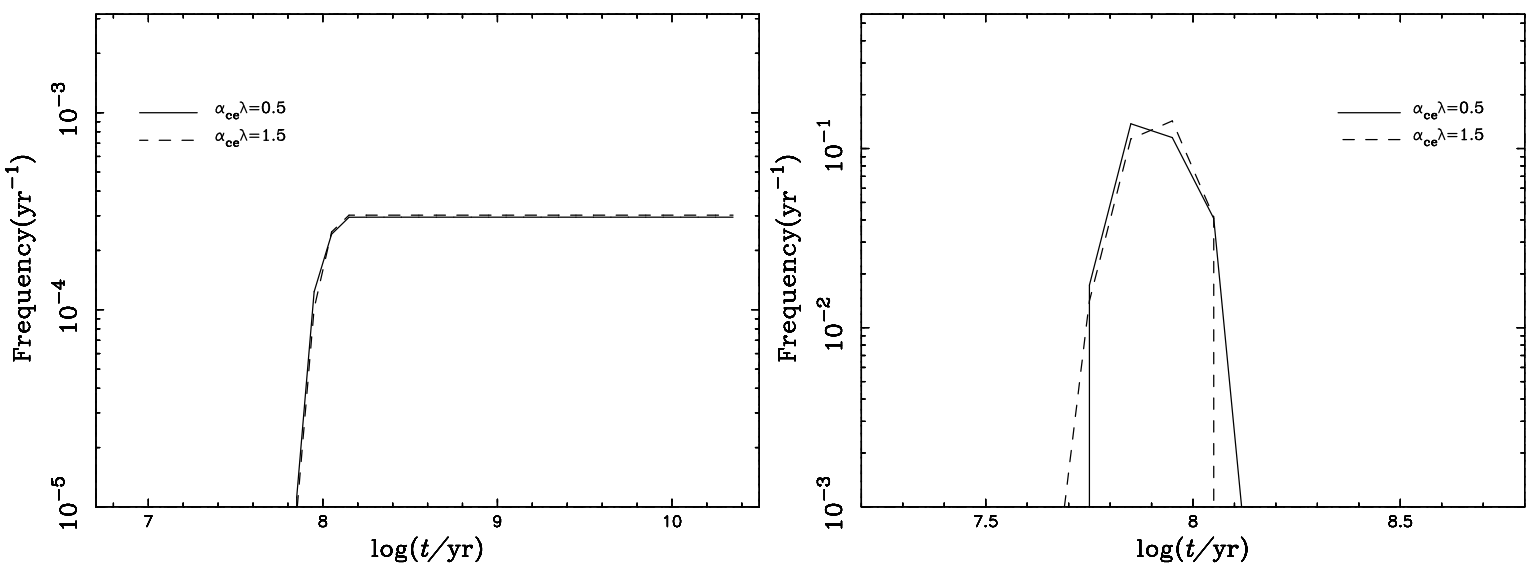

FIGURE 2. The evolution of SN Ia birthrates with time for the He star donor channel. Left panel: the evolution of Galactic SN Ia birthrates for a constant Pop I SFR $\left(5 M_{\odot} \mathrm{yr}^{-1}\right)$, where the solid and dashed curves show the results of different CE ejection parameters with $\alpha_{\mathrm{ce}} \lambda=0.5$ (solid) and $\alpha_{\mathrm{ce}} \lambda=1.5$ (dashed), respectively. Right panel: similar to the left panel, but for a single starburst with a total mass of $10^{11} M_{\odot}$. (From Wang et al. [48])
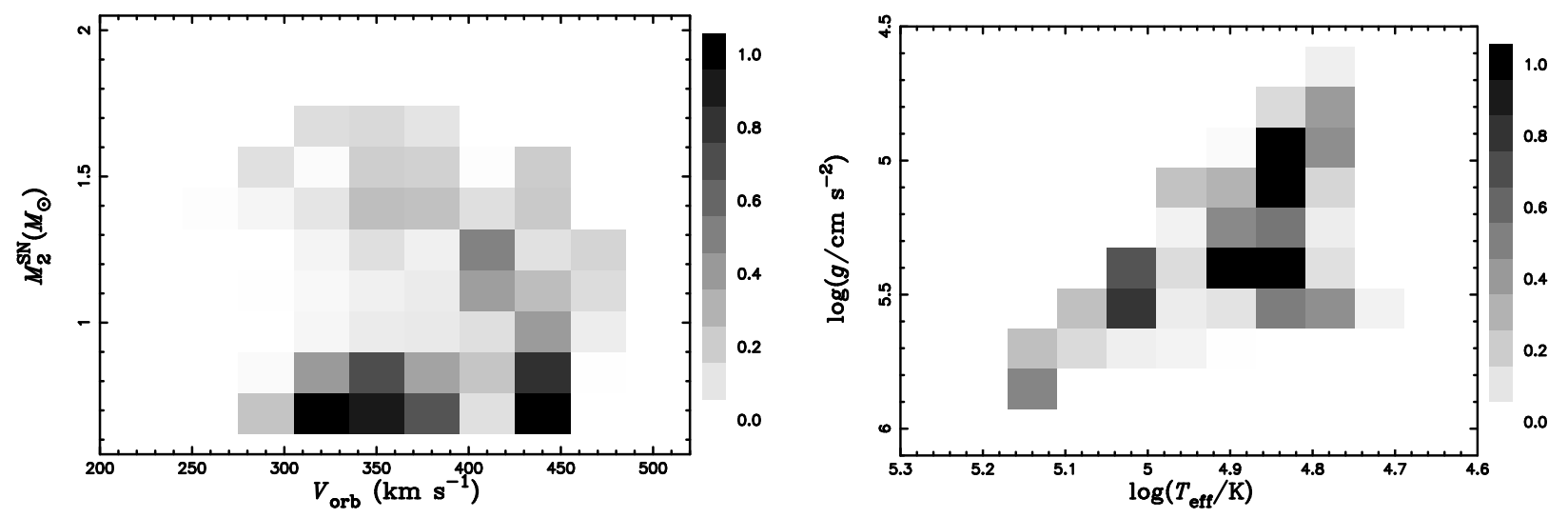

FIGURE 3. Left panel: the distribution of properties of companions in the plane of $\left(V_{\text {orb }}, M_{2}^{\mathrm{SN}}\right)$ at the current epoch, where $V_{\text {orb }}$ is the orbital velocity and $M_{2}^{\mathrm{SN}}$ the mass at the moment of SN explosion. Right panel: similar to the left panel, but in the plane of $\left(\log T_{\text {eff }}, \log g\right.$ ), where $T_{\text {eff }}$ is the effective temperature of companions and $\log g$ the surface gravity. (From Wang \& Han [7])
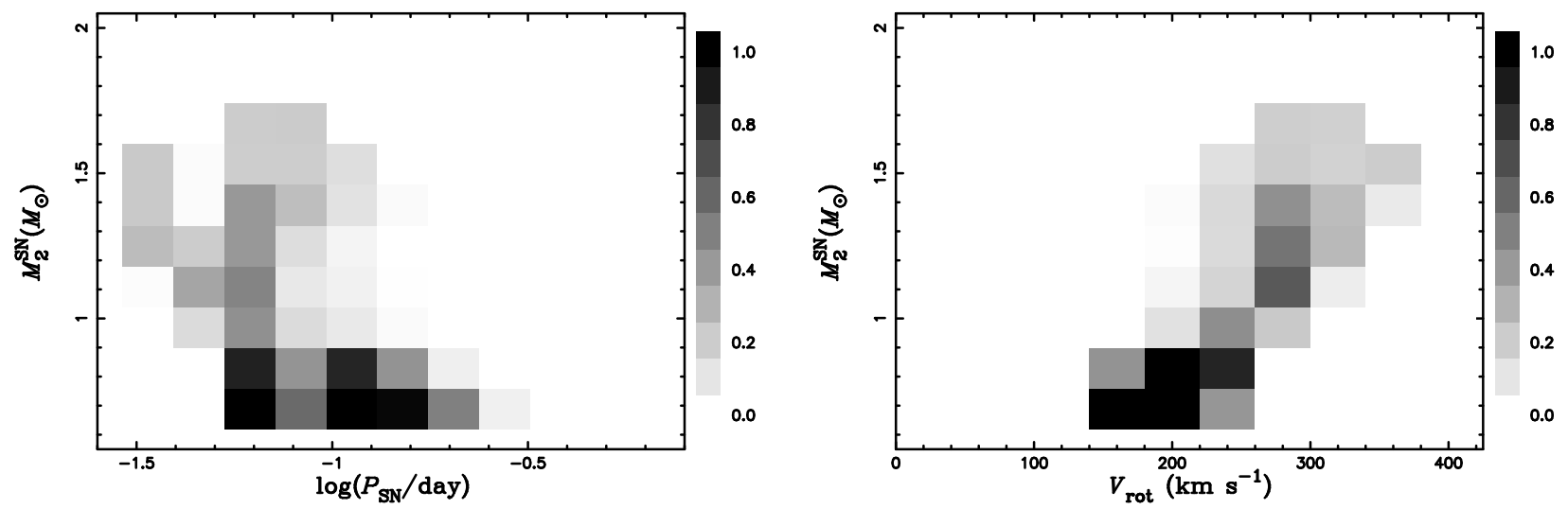

FIGURE 4. Left panel: similar to Fig. 3 , but in the plane of $\left(\log P^{\mathrm{SN}}, M_{2}^{\mathrm{SN}}\right)$, where $P^{\mathrm{SN}}$ is the orbital period. Right panel: similar to Fig. 3, but in the plane of $\left(V_{\text {rot }}, M_{2}^{\mathrm{SN}}\right)$, where $V_{\text {rot }}$ is the equatorial rotational velocity of companions. (From Wang \& Han [7]) 


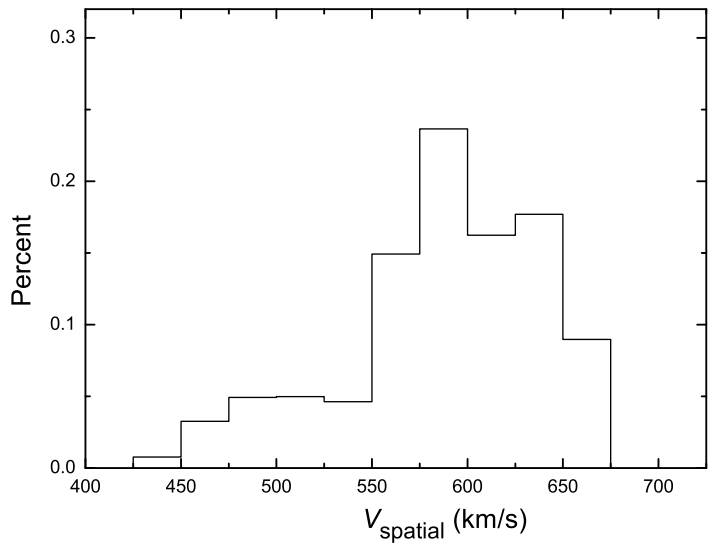

FIGURE 5. The distribution of the spatial velocity with $\alpha_{\mathrm{ce}} \lambda=0.5$. (From Wang $\&$ Han [7])

obtained the distributions of many properties of the surviving companions of this channel at the moment of SN explosion. Figs 3 and 4 shows the distributions of the masses, the orbital velocities, the effective temperatures, the surface gravities, the orbital period and the equatorial rotational velocity of companions at the moment of SN explosion (for the detailed discussion see [7]). These distributions may be helpful for identifying the surviving companions of $\mathrm{SNe}$ Ia. The simulation also shows the initial parameters of the primordial binaries and the WD + He star systems that lead to SNe Ia, which may help to search for potential SN Ia progenitors.

In Fig. 5, we show the current epoch distribution of the spatial velocity for the surviving companions from this channel (for details see [7]). We see that the surviving companions have high spatial velocities $(>400 \mathrm{~km} / \mathrm{s})$, which almost exceed the gravitational pull of the Galaxy nearby the Sun. Thus, the surviving companions from the SN explosion scenario could be an alternative origin for hypervelocity stars (HVSs), which are stars with a velocity so great that they are able to escape the gravitational pull of the Galaxy.

\section{DISCUSSION}

The simulations give Galactic SN Ia birthrate of $\sim 0.3 \times 10^{-3} \mathrm{yr}^{-1}$ for the He star donor channel, which is lower than that inferred observationally (i.e., $3-4 \times 10^{-3} \mathrm{yr}^{-1}$; Cappellaro and Turatto [52]). This implies that the He star donor channel is only a subclass of SN Ia production, and there may be some other channels or mechanisms also contributing to SNe Ia, e.g., WD + MS channel, WD + RG channel or double-degenerate channel (see [53]).

Hachisu et al. [54] recently studied the mass-stripping effect on a MS or slightly evolved companion star by winds from a mass-accreting WD. The model can also provide a possible ways of producing young SNe Ia, but the model depends on the efficiency of the mass-stripping effect. We also find that the model produces very few young SNe Ia according to a detailed BPS approach. Thus, we consider the He star donor channel as a main contribution to the formation of young SNe Ia.

Based on the optically thick wind assumption, Wang \& Han [55] recently calculated about $10000 \mathrm{WD}+\mathrm{He}$ star systems and obtained SN Ia production regions of the He star donor channel with different metallicities. For a constant star-formation galaxy, they found that SN Ia birthrates increase with metallicity. If a single starburst is assumed, $\mathrm{SNe}$ Ia occur systemically earlier and the peak value of the birthrate is larger for a high $Z$. We also note that Liu et al. [56] recently investigated the effects of rapid differential rotation on the accreting WD, and found that the highest mass of the accreting WD at the moment of SN Ia explosion is $1.81 M_{\odot}$ for the He star donor channel, which may provide a way for the formation of super-Ch mass SNe Ia in the observations.

Massive WD + He star systems are candidates of SN Ia progenitors. HD 49798/RX J0648.0-4418 is an evidence of the existence of massive WD + He star systems. Based on the data from the XMM-Newton satellite, Mereghetti et al. [57] recently derived the masses of the two components. The corresponding masses are $1.50 \pm 0.05 M_{\odot}$ for HD 

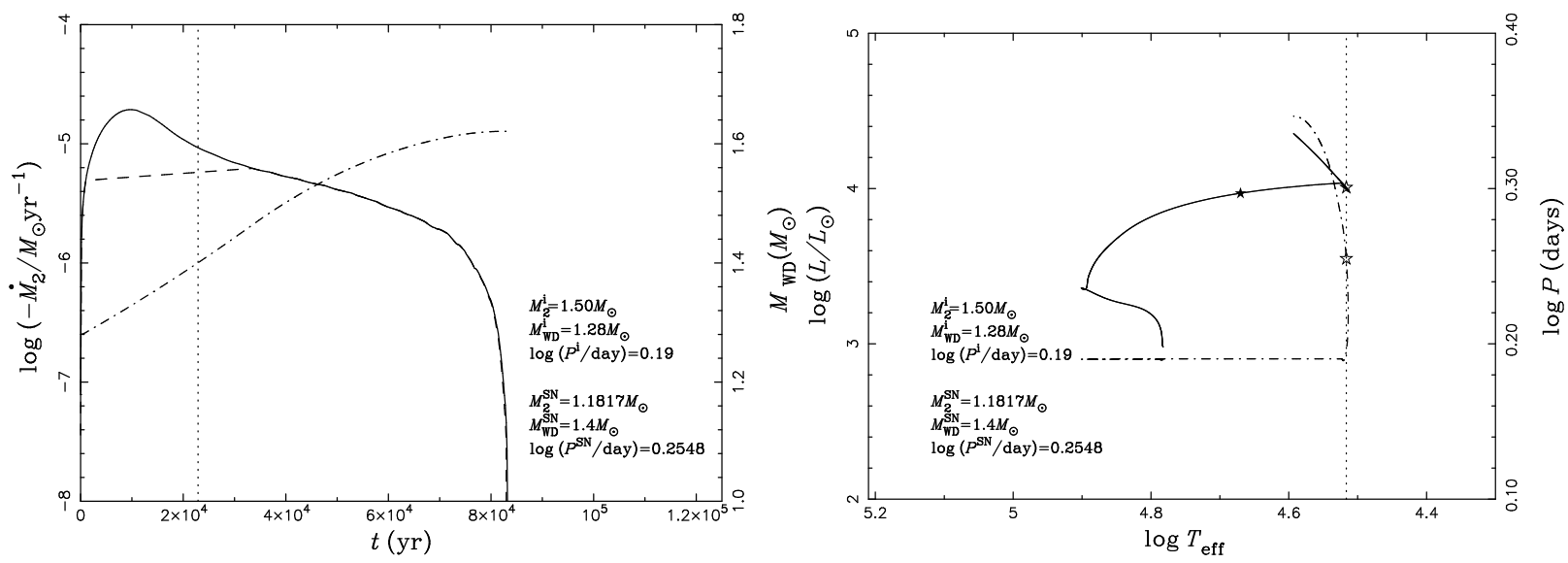

FIGURE 6. Results of binary evolution calculations with initial masses of two components and the orbital period as well similar to the binary system HD49798/RX J0648.9-4418. In the left panel, the solid, dashed and dash-dotted curves show the mass-transfer rate, the mass-growth rate of the WD and the mass of the WD varying with time after HD 49798 fills its Roche lobe, respectively. In the right panel, the evolutionary track of the He donor star is shown as a solid curve, and the evolution of the orbital period is shown as a dash-dotted curve. The solid star represents the current position of HD 49798. Dotted vertical lines in these two panels and open stars in the right panel indicate the position where the WD is expected to explode as an SN Ia. (From Wang \& Han [58])

49798 and $1.28 \pm 0.05 M_{\odot}$ for the WD. According to a detailed binary evolution model, Wang \& Han [58] found that the massive WD can increase its mass to the Ch mass in future evolution (see Fig. 6). Thus, HD 49798 with its WD companion is a likely candidate of SN Ia progenitors. V445 Pup is another candidate of massive WD + He star system, in which the mass of the WD is more than $1.35 M_{\odot}$, and the mass of the He star is more than $0.85 M_{\odot}[59,60]$. However, we still do not know the orbital period of the binary system and the mass of the He donor star so far. This needs further observations of V445 Pup after the dense dust shell disappears.

US 708 is an extremely He-rich sdO star in the Galaxy halo, with a heliocentric radial velocity of $+708 \pm 15 \mathrm{~km} / \mathrm{s}$ (Hirsch et al. [61]). We note that the local velocity relative to the Galatic center may lead to a higher observation velocity for the surviving companions, but this may also lead to a lower observation velocity. Considering the local velocity nearby the Sun $(\sim 220 \mathrm{~km} / \mathrm{s})$, we find that $\sim 30 \%$ of the surviving companions may be observed to have velocity $V>700 \mathrm{~km} / \mathrm{s}$ for a given SN ejecta velocity $13500 \mathrm{~km} / \mathrm{s}$. In addition, the asymmetric explosion of SNe Ia may also enhance the velocity of the surviving companions. Thus, a surviving companion in the He star donor channel may have a high velocity like US 708. In future investigations, we will employ the Large sky Area Multi-Object fiber Spectral Telescope (LAMOST) to search the HVSs originating from the surviving companions of SNe Ia.

\section{ACKNOWLEDGMENTS}

This work is supported by the National Natural Science Foundation of China (Grant Nos. 10821061 and 11033008), the National Basic Research Program of China (Grant No. 2007CB815406) and the Chinese Academy of Sciences (Grant No. KJCX2-YW-T24).

\section{REFERENCES}

1. Riess, A., Filippenko, A. V., Challis, P., et al. 1998, AJ, 116, 1009

2. Perlmutter, S., Aldering, G., Goldhaber, G., et al. 1999, ApJ, 517, 565

3. Hillebrandt, W., \& Niemeyer, J. C. 2000, ARA\&A, 38, 191

4. Wang, B., Meng, X., Wang, X.-F., \& Han, Z. 2008, ChJAA (Chin. J. Astro. Astrophys.), 8, 71

5. Podsiadlowski, Ph. 2010, Astron. Nachr., 331, 218

6. Greggio, L. 2010, MNRAS, 406, 22

7. Wang, B., \& Han, Z. 2009, A\&A, 508, L27

8. Wang, B., \& Han, Z. 2010, MNRAS, 404, L84

9. Meng, X., \& Yang, W. 2010, MNRAS, 401, 1118 
10. Webbink, R. F. 1984, ApJ, 277, 355

11. Iben, I., \& Tutukov, A. V. 1984, ApJS, 54, 335

12. Han, Z. 1998, MNRAS, 296, 1019

13. Nomoto, K., \& Iben, I. 1985, ApJ, 297, 531

14. Hachisu, I., Kato, M., \& Nomoto, K. 1996, ApJ, 470, L97

15. Li, X.-D., \& van den Heuvel, E. P. J. 1997, A\&A, 322, L9

16. Langer, N., Deutschmann, A., Wellstein, S., \& Höflich, P. 2000, A\&A, 362, 1046

17. Han, Z., \& Podsiadlowski, Ph. 2004, MNRAS, 350, 1301

18. Han, Z., \& Podsiadlowski, Ph. 2006, MNRAS, 368, 1095

19. Chen, W.-C., \& Li, X.-D. 2007, ApJ, 658, L51

20. Chen, W.-C., \& Li, X.-D. 2009, ApJ, 702, 686

21. Ruiter, A. J., Belczynski, K., \& Fryer, C. L. 2009, ApJ, 699, 2026

22. Lü, G., Zhu, C., Wang, Z., \& Wang, N. 2009, MNRAS, 396, 1086

23. Wang, B., \& Han, Z. 2010, RAA (Res. Astron. Astrophys.), 10, 235

24. Wang, B., Li, X.-D., \& Han, Z. 2010, MNRAS, 401, 2729

25. Meng, X., \& Yang, W. 2010b, ApJ, 710, 1310

26. Mennekens, N., Vanbeveren, D., De Greve, J. P., et al. 2010, A\&A, 515, A89

27. Hansen, B. M. S. 2003, ApJ, 582, 915

28. Voss, R., \& Nelemans, G. 2008, Nature, 451, 802

29. Wang, X.-F., Li, W.-D., Filippenko, A. V., et al. 2008, ApJ, 675, 626

30. Justham, S., Wolf, C., Podsiadlowski, Ph., \& Han, Z. 2009, A\&A, 493, 1081

31. Yoon, S.-C., \& Langer, N. 2003, A\&A, 412, L53

32. Mannucci, F., Della Valle, M., \& Panagia, N. 2006, MNRAS, 370, 773

33. Aubourg, E., Tojeiro, R., Jimenez, R., et al. 2008, A\&A, 492, 631

34. Brandt, T. D., Tojeiro, R., Aubourg, E., Heavens, A., et al. 2010, AJ, 140, 804

35. Maoz, D., Sharon, K., \& Gal-Yam, A. 2010, ApJ, in press [arXiv:1006.3576]

36. Eggleton, P. P. 1971, MNRAS, 151, 351

37. Eggleton, P. P. 1972, MNRAS, 156, 361

38. Eggleton, P. P. 1973, MNRAS, 163, 279

39. Han, Z., Podsiadlowski, Ph., \& Eggleton, P. P. 1994, MNRAS, 270, 121

40. Pols, O. R., Tout, C. A., Eggleton, P. P., \& Han, Z. 1995, MNRAS, 274, 964

41. Pols, O. R., Schröder, K. P., Hurly, J. R., et al. 1998, MNRAS, 298, 525

42. Han, Z., Tout, C. A., \& Eggleton, P. P. 2000, MNRAS, 319, 215

43. Chen, X., \& Tout, C. A. 2007, ChJAA (Chin. J. Astro. Astrophys.), 7, 245

44. Kato, M., \& Hachisu, I. 2004, ApJ, 613, L129

45. Wang, B., Meng, X., Chen, X., \& Han, Z. 2009a, MNRAS, 395, 847

46. Hurley, J. R., Pols, O. R., \& Tout, C. A. 2000, MNRAS, 315, 543

47. Hurley, J. R., Tout, C. A., \& Pols, O. R. 2002, MNRAS, 329, 897

48. Wang, B., Chen, X., Meng, X., \& Han, Z. 2009b, ApJ, 701, 1540

49. Miller, G. E., \& Scalo, J. M. 1979, ApJS, 41, 513

50. Wang, B., \& Han, Z. 2010, Astrophys. Space Sci., in press [arXiv:0911.4998]

51. Pan, K.-C., Ricker, P. M., \& Taam, R. E. 2010, ApJ, 715, 78

52. Cappellaro, E., \& Turatto, M. 1997, in Ruiz-Lapuente P., Cannal R., Isern J., eds, Thermonuclear Supernovae. Kluwer, Dordrecht, P. 77

53. Wang, B., Liu, Z., Han, Y., et al. 2010, ScChG (Sci. China Ser. G), 53, 586

54. Hachisu, I., Kato, M., \& Nomoto, K. 2008, ApJ, 679, 1390

55. Wang, B., \& Han, Z. 2010, A\&A, 515, A88

56. Liu, W.-M., Chen, W.-C., Wang, B., \& Han, Z. 2010, A\&A, in press [arXiv:1007.4751]

57. Mereghetti, S., Tiengo, A., Esposito, P., et al. 2009, Science, 325, 1222

58. Wang, B., \& Han, Z. 2010, RAA (Res. Astron. Astrophys.), 10, 681

59. Kato, M., Hachisu I., Kiyota S., \& Saio, H. 2008, ApJ, 684, 1366

60. Woudt, P. A., Steeghs, D., Karovska, M., et al. 2009, ApJ, 706, 738

61. Hirsch, H. A., Heber, U., O’Toole, S. J., \& Bresolin, F. 2005, A\&A, 444, L61 